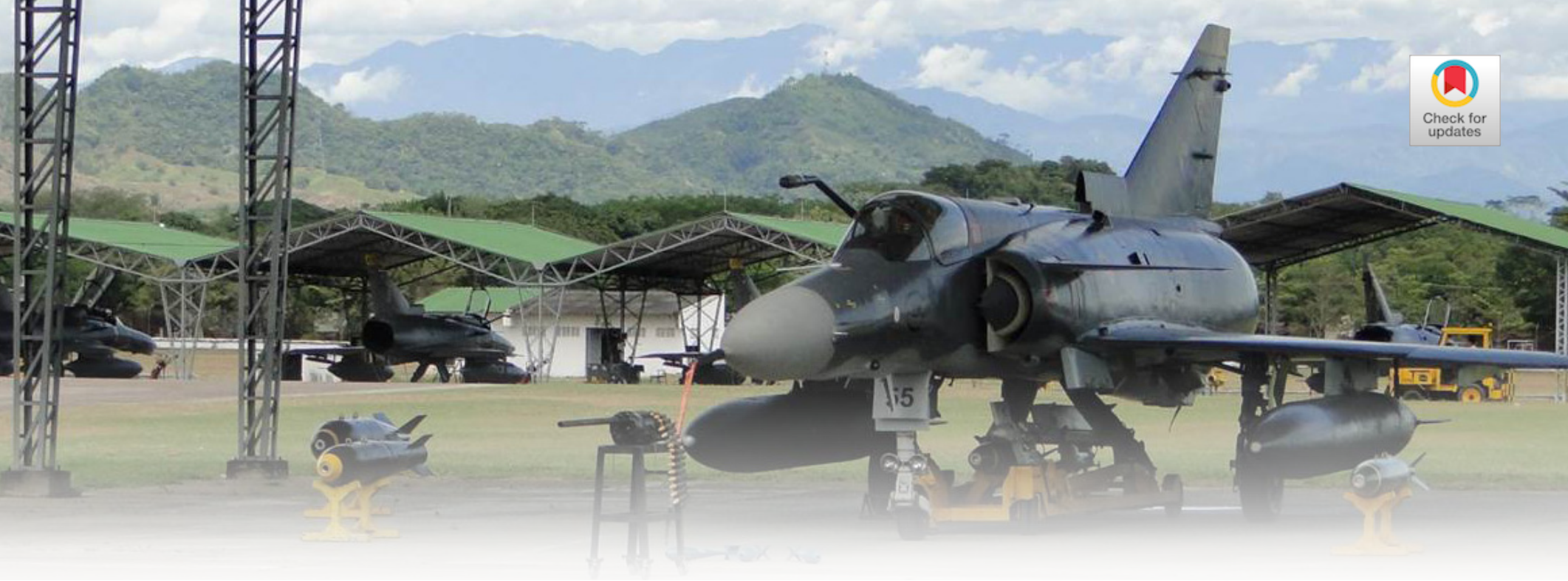

\title{
Diagnóstico del proceso de adquisiciones logísticas aeronáuticas a través del Portal de Proveedores de la Agencia de Compras FAC
}

\author{
An Assessment of the Process of Aeronautical Logistics Acquisitions \\ by the Supplier Portal of the Colombian Air Force's (FAC) Purchasing Agency ${ }^{2}$
}

Sonia María Peña Rendón ${ }^{3}$ Erika Juliana Estrada Villa ${ }^{4}$

CIENCIA Y PODER AÉREO ISSN 1909-7050 | E-ISSN 2389-2468 |Volumen 9 | Enero-Diciembre de 2014 | Colombia | Pp. 43-53

Recibido: 06/05/2013

Aprobado evaluador interno: 21/06/2013 Aprobado evaluador externo: 05/08/2013

Artículo científico original, que corresponde al trabajo investigativo titulado: Propuesta de mejora al proceso de adquisiciones logísticas aeronáuticas a través del portal de proveedores de la agencia de compras FAC, desarrollado por la MY. Sonia María Peña Rendón, dentro la Especialización en Logística Aeronáutica, Escuela de Postgrados de la Fuerza Aérea Colombiana.

Original scientific article, according to research entitled "Proposal for the Improvement of the Process of Logistical Aeronautic Procurement by the Supplier Portal of the FAC Purchasing Agency," developed by Sonia María Peña Rendón, as part of the Specialization in Aeronautical Logistics, Colombian Air Force's Graduate School.

${ }^{3}$ Oficial Fuerza Aérea Colombiana. Especialista en Logística Aeronáutica. Correo electrónico: soma-pe@hotmail.com Official Forces Air Colombian. Specialist in Aeronautical Logistics. E-mail:soma-pe@hotmail.com

${ }^{4}$ Docente, investigadora y asesora de trabajos de grado de investigación, Departamento de Investigación de la Escuela de Postgrados Fuerza Aérea Colombiana. Msc. Informática Educativa. Correo electrónico: ejeinvestigacion@gmail.com Professor, researcher and adviser of works of degree of research, Department of Researcher. McS. Educational Computer Science. E-mail:ejeinvestigacion@gmail.com
Resumen: La propuesta de mejora al Portal de Proveedores del Exterior de la Fuerza Aérea Colombiana, es un trabajo de investigación efectuado con el fin de fortalecer los canales de comunicación y reducir el tiempo de repuesta en el proceso logístico de abastecimientos, agilizando y optimizando la herramienta de adquisiciones de la Agencia de Compras de la FAC, de tal manera que se garantice la credibilidad del sistema ante los proveedores y la transparencia en el uso de la información. El artículo se enfocó en determinar las falencias del sistema, mediante la evaluación del Portal por parte de proveedores y usuarios internos, así como el análisis a la documentación sobre novedades del sistema, arrojando como resultado que las principales problemáticas en el portal de proveedores se derivan de la falta de conocimiento en el manejo de la página web y la funcionalidad del sistema por ausencia de transacciones que permiten realizar operaciones en las cotizaciones.

Palabras clave: Adquisiciones, cadena de abastecimiento, proceso logístico, portal de proveedores.

Abstract: This investigative project proposes improvements in the Colombian Air Force's (FAC) Foreign Supplier Portal, with the aim of strengthening communication channels and reducing response time in the supplies logistical process. By streamlining and optimizing the FAC's acquisition tool, the system's credibility with suppliers will be ensured, as will transparency in the use of information. The article focused on identifying the weaknesses of the system through an assessment of the portal from the perspective of suppliers and internal users, as well as by an analysis of the new documentation system. It asserts that the chief problems derive from a lack of knowledge in using the web platform, and the performance of the system because of a lack of transactions that would allow budget operations.

Key Words: Procurement, Logistical Process, Supplier Portal, Supply Chain. 


\section{Introducción}

El presente artículo se enfoca en presentar una propuesta de mejora, para el proceso de compras de la Jefatura de Operaciones Logísticas de la Fuerza Aérea Colombiana (FAC); específicamente abordando las novedades surgidas en la utilización de las herramienta informática con la cual cuenta la Agencia de Compras, para adelantar sus procesos de adquisiciones logísticas, denominada Portal de Proveedores; que corresponde a una página web en la cual las empresas inscritas como proveedores en ACOFA pueden consultar en tiempo real los procesos precontractuales y contractuales publicados, siendo un desarrollo efectuado en la plataforma del sistema SAP implementado desde 2010, para utilizar la información logística de mantenimiento y del módulo financiero. Las novedades presentadas en la utilización del sistema impactan significativamente la gestión de materiales, por lo tanto, se requiere diagnóstico y planteamiento de posibles soluciones, teniendo en cuenta que el sistema requiere ajustes para su optimización.

El mejoramiento al Portal de Proveedores es una herramienta innovadora en el sector Defensa, para realizar los procesos logísticos aeronáuticos, refleja la importancia que tienen en la FAC los procesos de gestión de materiales realizados a través de la Agencia de Compras, correspondiendo dichas adquisiciones al $74 \%$ del presupuesto de funcionamiento e inversión de la Institución, lo que conlleva a que la Jefatura de Operaciones Logísticas busque un mecanismo que permita satisfacer oportunamente la cadena de abastecimientos que impacta directamente a los requerimientos aeronáuticos y de equipo asociado de las Unidades Aéreas, incidiendo directamente en el alistamiento aeronáutico y garantizando a la Jefatura de Operaciones Aéreas contar con los medios que permitan el cumplimiento de la misión institucional. Así mismo, al optimizar el sistema de compras de la Agencia de Compras donde se ejecuta gran cantidad de recursos del presupuesto público, se da cumplimiento al cuarto objetivo estratégico de la Fuerza Aérea; el cual corresponde a: ser una organización administrada con excelencia, en concordancia con las políticas Institucionales referentes a la administración de la información, simplificación y racionalización, tanto de procesos, como de procedimientos.

El objetivo general de la investigación es elaborar una propuesta de mejora al Portal de Proveedores del Exterior, basada en la evaluación efectuada al sistema por parte de los usuarios, tanto internos, como exter- nos y la recopilación de la información documental; a fin de optimizar el proceso de adquisiciones logísticas aeronáuticas a través del Portal de Proveedores, para recopilar la información, se dividieron los grupos de usuarios, de acuerdo a la experiencia en el manejo del portal, permitiendo evidenciar la problemática, la cual trae como consecuencia demoras y/o traumatismos en la gestión logística de materiales, impactando directamente el alistamiento de aeronaves, al no contar con un canal de adquisiciones óptimo y oportuno.

Como usuarios directos, se encuentra el personal de la Subdirección de Adquisiciones de ACOFA, dependencia encargada de realizar el proceso precontractual de la Agencia de Compras y como usuarios indirectos la Dirección de Procesos Logísticos, la cual realiza el proceso de selección y adjudicación de los procesos de adquisición de repuestos y servicios. Otro usuario indirecto es la Dirección de Mantenimiento e Ingeniería, esta realiza el proceso de adjudicación de equipos de aviónica, herramientas y equipo ETTA principalmente.

El alcance del presente trabajo está dado por la necesidad manifiesta de la Subdirección de Adquisiciones de ACOFA de la Jefatura de Operaciones Logísticas, para realizar en forma objetiva y estructurada mejoras al Portal de Proveedores del Exterior, a fin de enlazar el proceso de adquisiciones logísticas, convirtiendo la herramienta en pilar fundamental en la cadena de abastecimientos.

\section{Método}

El desarrollo del presente trabajo se llevó a cabo con tres tipos de investigación de acuerdo con las fases adelantadas, las cuales son: descriptiva, evaluativa y documental; La investigación evaluativa utiliza el método cuantitativo, por la matriz de Krajlic (M4P) revisada por (Pérez, 2012) que permite determinar la segmentación de los bienes a adquirir y tipo de proveedores de acuerdo a la complejidad del mercado, así mismo es una investigación mixta al aplicar encuestas que observan factores cualitativos. Por otro lado, la investigación descriptiva definirá las características del sistema buscando resolver la pregunta de cómo está estructurado el programa y cuáles son las partes interrelacionadas, la investigación evaluativa permitirá, mediante información sistemática y agrupada, seleccionar los principales problemas a solucionar utilizando herramientas Estadísticas, para que de manera objetiva se encauce el planteamiento del problema (Hernández, y otros, 2011). 
Para definir el diseño metodológico se implementó la aplicación de encuestas estructuradas sobre el funcionamiento del sistema, estas se aplicaron a las empresas registradas como proveedores. El instrumento requirió la división de grupos de usuarios de acuerdo al conocimiento y nivel de experticia en el manejo de la página web y la recopilación de la información por correo electrónico. Posteriormente, se realizó el análisis de los datos mediante diagramas de afinidad, para determinar los problemas evidenciados que presentan mayor impacto en el desarrollo del proceso y que suceden con mayor frecuencia; finalmente, se recurre a la investigación documental y descriptiva para medición de factores cualitativos; ya que debido a su subjetividad requieren ser evaluados en forma diferente.

Con el fin de desarrollar encuestas de evaluación a los usuarios del sistema que a mayo de 2012 eran 152 proveedores registrados en el portal, como usuarios activos, fue necesario dividir la población en dos grandes grupos, en primer lugar, los proveedores con mayor manejo o pericia en el sistema, quienes equivalen a 82 usuarios y en segundo lugar los proveedores que tienen una baja frecuencia de manejo del portal y que por el tipo de bienes que ofrecen no es rutinario su ingreso a la herramienta, quienes equivalen a 70 usuarios, para lograr la división de proveedores se requiere segmentar y establecer grupos homogéneos, aplicando la encuesta al 100\% de los proveedores. Dentro de la investigación se utilizó la totalidad de la población, es decir no hay selección de la muestra, ya que se aplicó un censo de la misma también conocido como estudio total.

De igual manera, las evaluaciones permitieron obtener y analizar datos cuantificables sobre variables y correlación entre las mismas, con el fin de generalizar y determinar una serie de objetivos específicos, obteniendo un alto grado de objetividad mediante la aplicación de métodos estadísticos tradicionales, enfocando el desarrollo de la investigación en plantear mejoras a las situaciones que suceden con mayor frecuencia y que causan mayor impacto en la función del Portal. Otro factor implícito en la metodología fue la revisión y análisis de los resultados de las auditorías realizadas al proceso logístico de adquisiciones en forma interna, así como las adelantadas por entes de control, buscando detectar observaciones o hallazgos concernientes a la gestión de materiales, sin encontrar en este caso antecedentes de emisión de informes sobre el tema de la investigación.
Para abordar la investigación documental se analizó la Directiva 004 de febrero de 2012 emitida por la Jefatura de Operaciones Logísticas, en la Subdirección de Adquisiciones que trata de las actividades a desarrollar para la adquisición de bienes y servicios a través de la Agencia de Compras de la FAC, con sede en Fort Lauderdale, Florida - Estados Unidos de América, confrontando si los procesos actuales que efectúa el Portal de Proveedores se encuentran de acuerdo con la directriz Institucional, determinando como resultado que los procesos de cotización, estudios de mercado y registro de proveedores, están acordes con lo establecido normativamente, sin embargo, la funcionalidad del sistema que ofrece sobre el cargue de novedades en el recibo de material por parte de la Dirección de Comercio Exterior no se encuentra parametrizada en la Directiva. No obstante este factor no afecta el proceso precontractual del sistema.

Adicionalmente, se indagaron fuentes jurídicas, sobre la existencia de otras fuentes legales para el manejo contractual de la Agencia de Compras, pero en Colombia no se encuentra legislación para este aspecto, por lo tanto se debe dar cumplimiento al Estatuto Anticorrupción, principios de contratación emanados de la Ley 80 de 1993, desarrollados en el marco legal y la observancia a la responsabilidad del servidor público en el manejo de recursos del patrimonio público sin importar su ejecución en el exterior.

En cuanto a las Leyes sobre negocios comerciales del Estado de la Florida de Estados Unidos, mediante las cuales se interpretan las relaciones: comprador (Agencia de Compras) y vendedor (cualquier proveedor del exterior), de acuerdo a la suscripción del contrato, se establecen las obligaciones y derechos de las partes, siendo importante el cumplimiento de los compromisos adquiridos y el principio de la "buena fé" en la legalidad de los documentos allegados por el proveedor. Con respecto al proceso de adjudicación o precontractual no existen lineamientos jurídicos específicos en el Estado de la Florida (Peña, 2012).

Referente a informes, comunicaciones internas y externas sobre el funcionamiento del Portal de Proveedores se recopilaron 41 documentos, entre informes y comunicaciones enviadas a la Subdirección de Adquisiciones relacionadas al Portal de Proveedores en el período comprendido entre enero de 2011 a mayo de 2012; debido a que al analizar detalladamente estos documentos, no se observa en forma objetiva la proble- 
mática manifestada sobre el funcionamiento del portal y teniendo en cuenta la ambigüedad de las comunicaciones y la combinación de factores precontractuales del sistema con inconformidades en la adjudicación, es necesario determinar en forma específica la causa en cada evento documentado.

Finalmente, después de realizar un análisis interpretativo de los resultados obtenidos, se analizaron las normas contractuales existentes que pueden aplicar al proceso de adquisiciones en el exterior por parte de la Fuerza Aérea, siendo consecuentes con los objetivos del Plan Estratégico Institucional 2011-2030 y teniendo en cuenta la complejidad de los procesos logísticos aeronáuticos de la Institución. Se evidenció la dificultad en la consecución de repuestos aeronáuticos para algunos de los 48 sistemas logísticos con los que cuenta actualmente la FAC, debido a la falta de producción aeronáutica nacional, situación que no permite satisfacer las necesidades de componentes aeronáuticos en Colombia. Así mismo, se determinó la necesidad de un mayor cuerpo de trabajo en el área de Adquisiciones.

\section{Definiciones abordadas}

La investigación se enmarca en un acercamiento conceptual desde la logística para determinar soluciones viables, técnicas, económicas de funcionalidad y capacitación para el usuario; contribuyendo a optimizar la cadena logística de abastecimientos, mediante la reducción en tiempo de respuesta en la consecución de la gestión de materiales, permitiendo atender necesidades de la FAC y de otras Fuerzas Militares de Colombia.

La logística de acuerdo con Ballou \& Ronald (2004), "es una ciencia auxiliar de la Administración definida como el conjunto de medios y métodos que permiten llevar a cabo la misión de una organización; implica tareas de planificación y gestión de recursos", su función es implementar y controlar con eficiencia los materiales y los productos desde el punto de origen, hasta el consumo, con el fin de satisfacer las necesidades en el menor tiempo posible. La logística empresarial, por medio de la administración logística y de la cadena de suministro, cubre tanto la gestión, como la planificación de las actividades de los departamentos de compras, producción, transporte, almacenaje y distribución.

El flujo logístico es particularmente importante en la fabricación just in time (justo a tiempo) en la cual el gran énfasis se pone en reducción al mínimo del stock.
Una tendencia reciente en grandes cadenas de distribución es asignar estas metas a los artículos comunes individuales, más que optimizar el sistema entero para un objetivo determinado. Esto es posible porque los planes describen generalmente las cantidades comunes que se almacenarán en cada localización y éstos varían dependiendo de la estrategia. El método básico de optimizar un sistema estándar de distribución, es utilizar un árbol de cobertura mínima de distribución para diseñar la red de transporte y después situar los nodos de almacenaje dimensionados para gestionar la demanda mínima, media o máxima de artículos. Frecuentemente, la demanda está limitada por la capacidad de transporte existente fuera de la localización del nodo de almacenaje tal como lo menciona Carranza (2004).

A partir de estas experiencias, la logística empresarial se encargó de estudiar la entrega de los bienes y servicios en lugar apropiado, en el momento preciso y bajo las condiciones adecuadas. Esto permite que las empresas cumplan con los requerimientos de sus clientes y obtengan la mayor rentabilidad posible. La logística aeronáutica en la FAC, busca mediante la integración y estandarización detallada de los procesos que hacen parte de la cadena logística, lograr: efectividad y eficiencia a través de tiempos de respuesta oportuna, niveles de disponibilidad de aeronaves y equipos asociados, sistemas de aseguramiento y control de calidad, niveles de reservas estratégicas de material aeronáutico, óptimos tiempos de nacionalización y distribución de material e integración de proveedores con alto grado de especialización.

Mediante la implementación y difusión del proceso de gestión de materiales FAC, en el sistema de gestión de calidad Kawak, la Jefatura de Operaciones Logísticas Aeronáuticas ha propiciado la política de mejoramiento continuo con alta visión estratégica y prospectiva. En el caso particular de la gestión del material aeronáutico, liderada por la Dirección de Control de Material Aeronáutico, se diseñaron dos estrategias: La primera consiste en la sincronización de los procesos logísticos críticos mediante la implementación de un proyecto de rediseño del Programa Anual de Soporte Logístico, PASL, con resultados decisivos en la estandarización de material para el Mantenimiento Programado, así como, el análisis de las 14 variables que hacen parte del Planeamiento Logístico Aeronáutico las cuales incluyen el diseño de escenarios según la capacidad instalada para producir horas de vuelo en los Grupos Técnicos y su análisis en las áreas logística y operacional, proyección del ciclo de 
vida de aeronaves, el análisis de la responsabilidad logística asignada a cada Unidad, entre otras.

La segunda estrategia hace referencia a que una vez diseñado el proyecto se implemente en todas las Unidades teniendo en cuenta los factores prioritarios; tales como consolidación, costos por equipo, estandarización, integración de pronósticos, definición de niveles de existencias para agotar reservas de material aeronáutico, con lo que se obtiene un alto impacto en aprovisionamiento y optimización del presupuesto mediante la identificación de material.

En comercio exterior se plantean necesidades de transporte más complejas como consecuencia de la distancia geográfica; las reglamentaciones aduaneras de los países; la necesidad de utilizar diferentes medios de transporte como el aéreo, terrestre, marítimo, férreo y multimodal con su respectivo proceso de almacenamiento. Las funciones de la Dirección de Comercio Exterior de acuerdo al mapa de procesos publicado en sistema de gestión de calidad, kawak que determina planificar y coordinar funciones relacionadas con la logística, como se observa en la Figura 1.

\section{Recepción de material aeronáutico proveniente del exterior, en cumplimiento de contratos con la Agencia de Compras de la Fuerza Aérea Colombiana, ACOFA.}

Recepción de material reparable y reacondicionamiento para el envío al exterior sin novedades de embalaje.

Ubicación en el exterior de material aeronáutico para actualización, mantenimiento, devolución, reparación y garantías.

Almacenaje y distribución de material aeronáutico a las diferentes Unidades Aéreas.

Realización de trámites de nacionalización del material recibido del exterior por compra o por reexportación.

Figura 1: Funciones la dirección de comercio exterior DICEX

Fuente: elaboración de las autoras, basado en SGC Kawak (2012).

\section{Análisis de los resultados}

Una vez obtenidos los datos se evidencia en asocio con su respectivo análisis los siguientes resultados:

En la Figura 2, se observa el resultado de la obtención de los datos evidenciados en la información documental agrupándolas por tipo de novedad, impacto en el proceso y la intensidad con la cual se presentan o frecuencia de repetición, con el fin de ilustrar su calificación se utiliza un diagrama de dispersión. La documentación que se refiere a fallas de conectividad, será descartada teniendo en cuenta que no será objeto del presente trabajo de investigación por no ser viable la mejora por parte de la FAC, debido a que la administración de los servidores del sistema se encuentra en el Ministerio de Defensa Nacional.

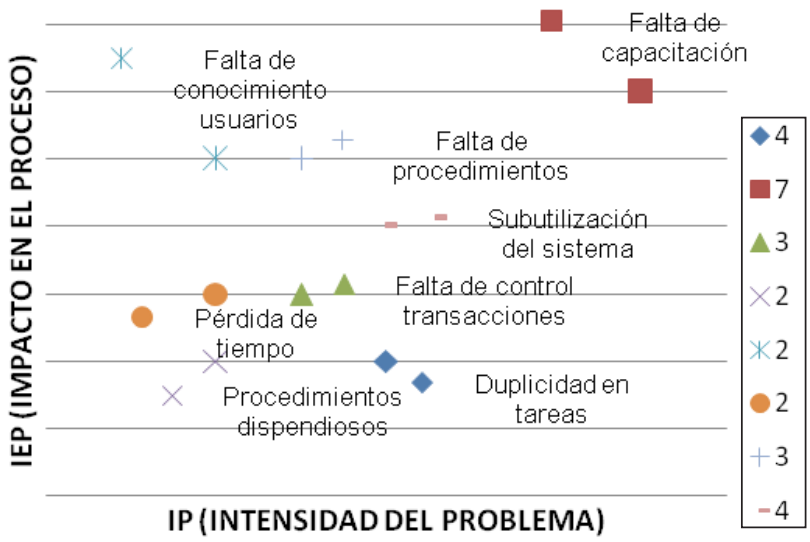

Figura 2. Diagrama del resultado obtención de información documental novedades portal de proveedores.

Fuente: Peña S. M. (2012). Propuesta de mejora al proceso de adquisiciones logísticas aeronáuticas a través del portal de proveedores de la agencia de compras FAC (p.64). Trabajo de grado para optar el título de Especialista en Logística Aeronáutica, Bogotá, Colombia: Instituto Militar Aeronáutico, Fuerza Aérea Colombiana,

En la Figura 3 se puede determinar el puntaje obtenido por los factores evaluados en la encuesta siendo considerado menor a 3,5 deficiente, de 3,6 a 4,0 como adecuado y de 4,1 a 5,0 sobresaliente. Los factores que obtuvieron calificación deficiente corresponden a retroalimentación de la información, actualización de la documentación legal, tiempo de validez de la oferta y capacitación de los proveedores.

En la Figura 4 se ilustran en forma de diagrama de barras los resultados de la encuesta modelo B, aplicada a los proveedores en las cuales se pueden establecer claramente los factores que según las percepciones de los usuarios afectan significativamente el funcionamiento del portal de proveedores; la obtención y procesamiento de datos permite consolidar y analizar la tabulación, para determinar posibles planes de acción.

Una vez obtenido los resultados evidenciados en las Figuras 3 y 4 se efectúa el comparativo entre los factores calificados como "Deficiente", es decir novedades que son observadas por los diferentes proveedores que presentan una calificación baja, de acuerdo a las encuestas analizadas. 


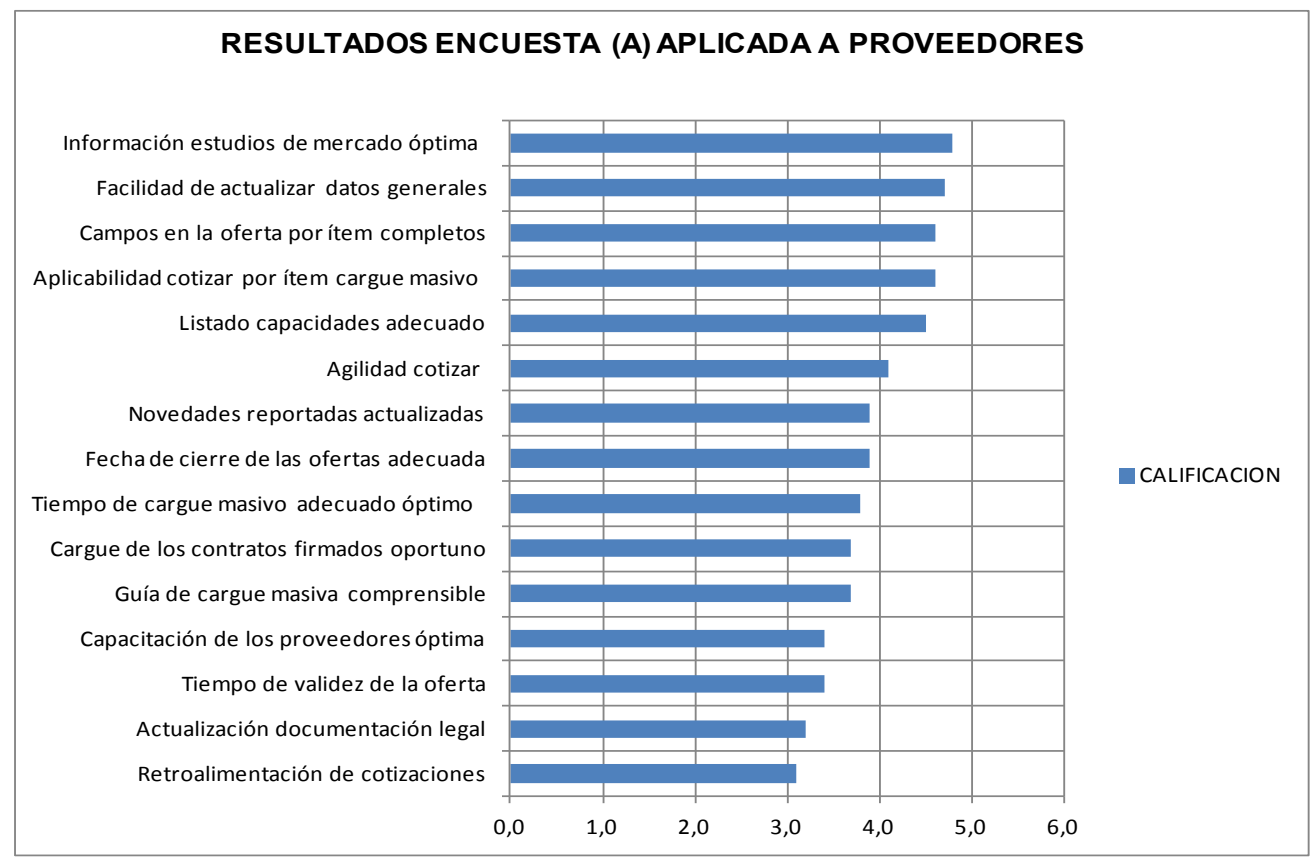

Figura 3. Resultados encuesta modelo (A) para determinar los factores que afectan el proceso de adquisiciones logísticas aeronáuticas a través del portal de proveedores de acuerdo a Tabla 3.

Fuente: Peña, S. M. (2012). Propuesta de mejora al proceso de adquisiciones logísticas aeronáuticas a través del portal de proveedores de la agencia de compras FAC (p.66). Trabajo de grado para optar el título de Especialista en Logística Aeronáutica. Bogotá, Colombia: Instituto Militar Aeronáutico, Fuerza Aérea Colombiana.

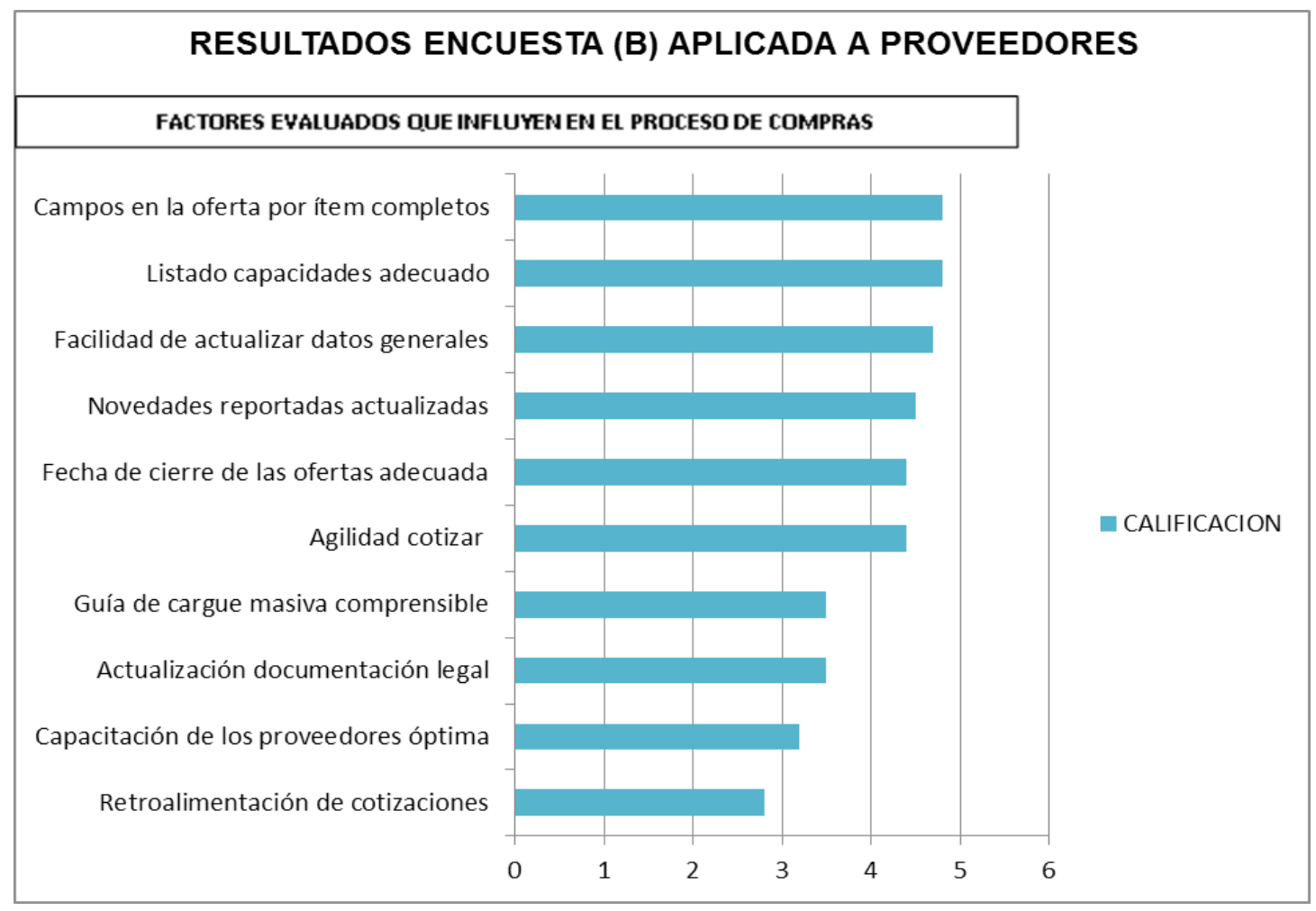

Figura 4. Resultados encuesta modelo (B) para determinar los factores que afectan el proceso de adquisiciones logísticas aeronáuticas a través del portal de proveedores de acuerdo a la tabla 4.

Fuente: Peña, S. M. (2012). Propuesta de mejora al proceso de adquisiciones logísticas aeronáuticas a través del portal de proveedores de la agencia de compras FAC (p.66) Trabajo de grado para optar el título de Especialista en Logística Aeronáutica. Bogotá, Colombia: Instituto Militar Aeronáutico, Fuerza Aérea Colombiana. 


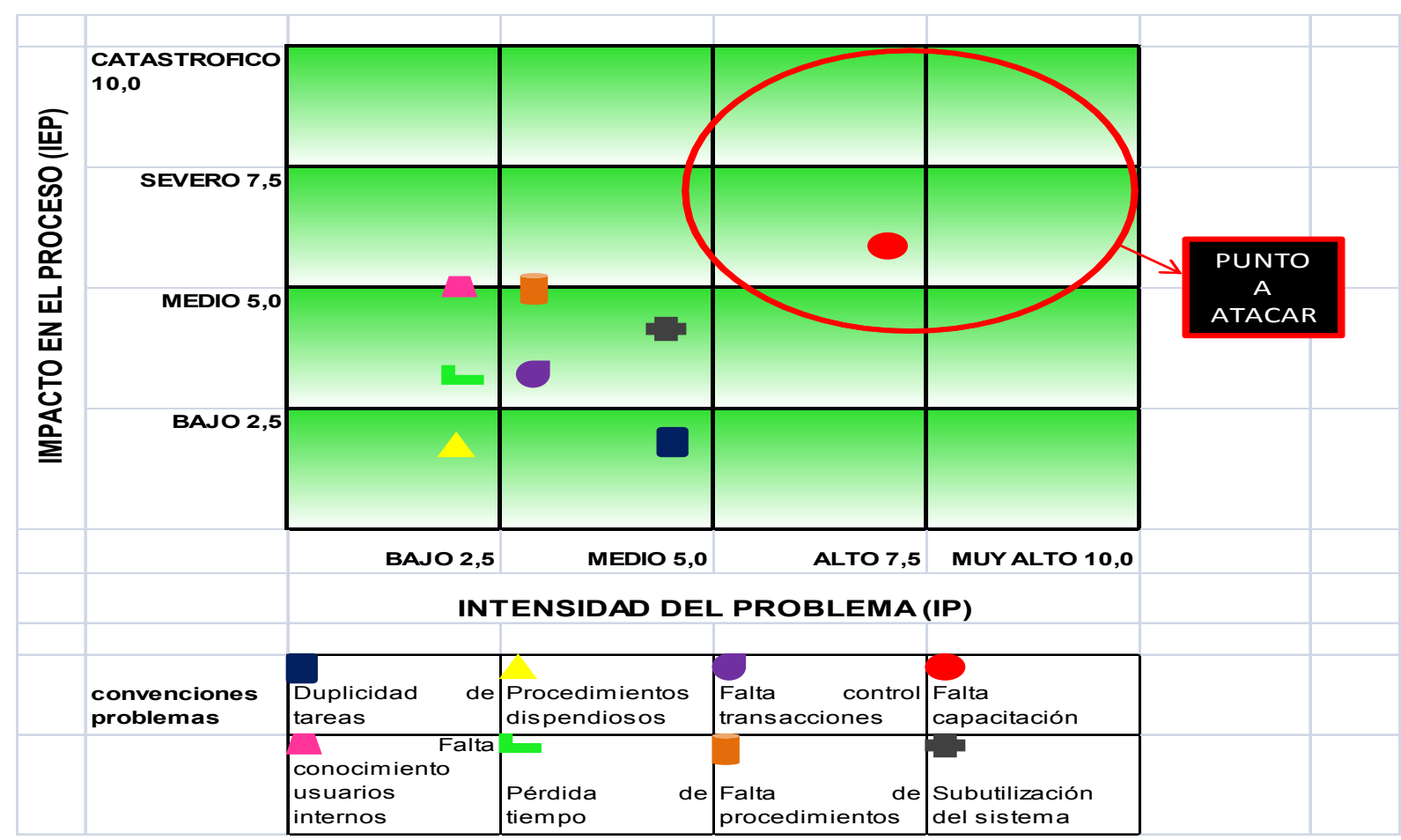

Figura 5. Diagrama Dispersión con los Factores Evaluados en el Portal de Proveedores por Usuarios Internos, que Afecta el Proceso de las Adquisiciones Logísticas.

Fuente: Peña S. M. (2012) Propuesta de mejora al proceso de adquisiciones logísticas aeronáuticas a través del portal de proveedores de la agencia de compras FAC (p.70). Trabajo de grado para optar el título de Especialista en Logística Aeronáutica, Bogotá, Colombia: Instituto Militar Aeronáutico, Fuerza Aérea Colombiana.

A continuación utilizando el diagrama de dispersión de la Figura 5, se localiza la calificación de la hoja de resumen M4P calificado, de manera que en forma determinada se puede definir la problemática con mayor grado de Intensidad del Proceso (IP) e Impacto en el proceso (IEP).

Fundamentándose en el diagrama de dispersión, el cual arroja estadísticamente los resultados o lo datos que permiten determinar los factores que deben ser atacados prioritariamente, para esta investigación se identifica el factor Falta de Capacitación, con el fin de mejorar el proceso de compras a través del portal de proveedores, mediante la determinación de los problemas que se presentan con mayor intensidad y que tiene mayor impacto en el proceso.

Con el fin de reflejar la frecuencia relativa de los problemas, se utiliza el diagrama de Pareto que se observa en la Figura 6, para analizar la problemática del portal de acuerdo a la investigación documental, donde se puede determinar las falencias más frecuentes.

Finalmente, como parte del desarrollo de las fases de investigación se analizaron los resultados obtenidos en auditorias al proceso de Logística Aeronáutica, por parte de la Inspección General de la FAC, referentes al proceso precontractual, como son hallazgos de no conformidades y oportunidades de mejoras, los cuales tenían relación directa o indirecta con el funcionamiento del Portal de Proveedores de los últimos dos años, tiempo promedio de existencia de la herramienta, pero no se encontró ningún tipo de observación al Portal, atribuible, tal vez, a lo incipiente del sistema y a la falta de conocimiento por parte del personal auditor sobre la página Web.

Así mismo, se revisaron los informes con enfoque integral presentados por la Contraloría General de la República (Ente de Control de las Entidades Públicas), correspondientes a las vigencias 2010 y el informe preliminar presentado en el mes de mayo de 2012 sobre la vigencia 2011, sin encontrar ninguna observación o recomendación sobre el tema tratado en el presente trabajo de investigación.

Con la investigación descriptiva, se determinan características comunes, se permite conceptuar sobre el estado actual del sistema, basándose en el trabajo obtenido en las fases anteriores y realizar en forma contundente la propuesta de mejora al sistema; atendien- 


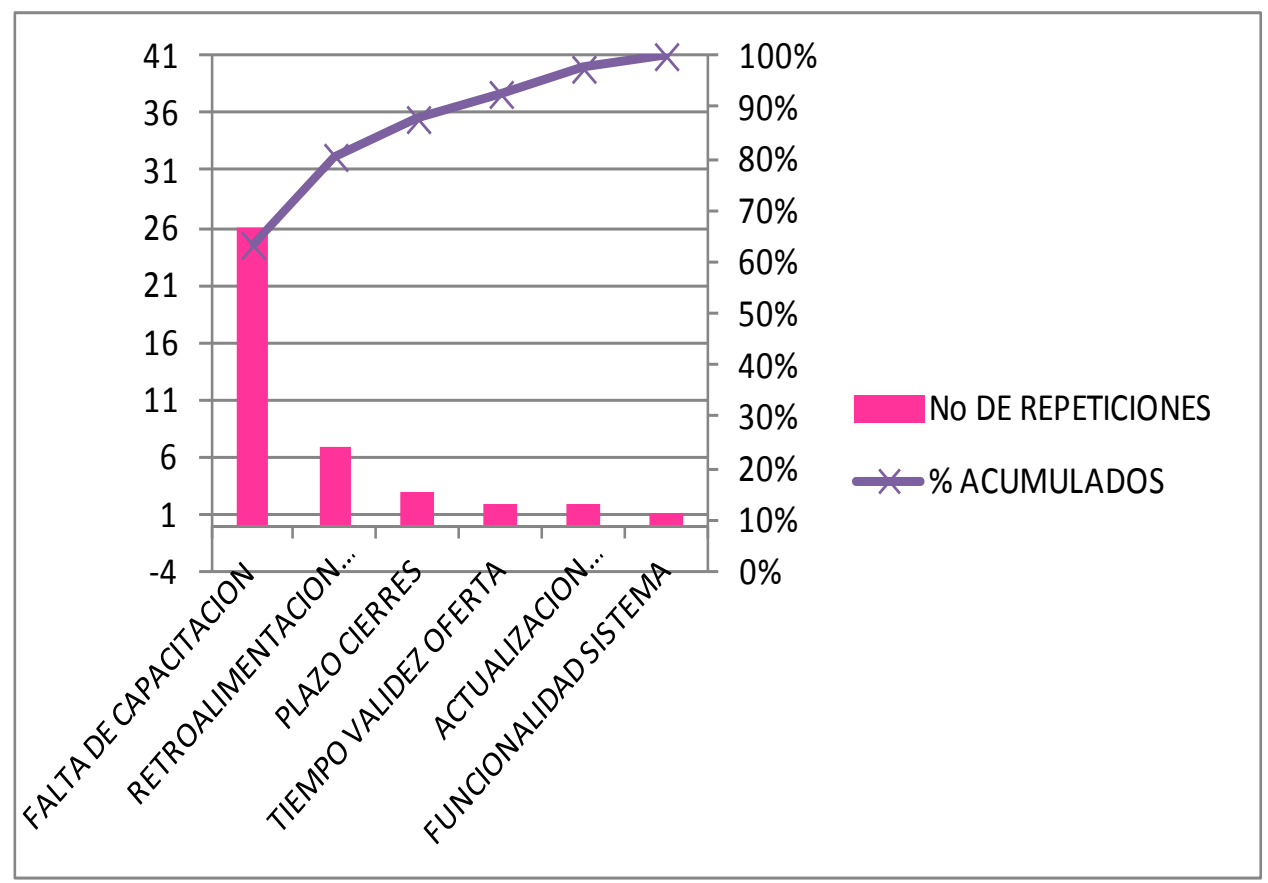

Figura 6. Diagrama de Pareto análisis de problemática portal de acuerdo con resultados investigación documental.

Fuente: Peña, S. M. (2012). Propuesta de mejora al proceso de adquisiciones logísticas aeronáuticas a través del portal de proveedores de la agencia de compras FAC (p.72) Trabajo de grado para optar el título de Especialista en Logística Aeronáutica, Bogotá, Colombia: Instituto Militar Aeronáutico, Fuerza Aérea Colombiana.

do los puntos más álgidos y que presentan una mayor relevancia e impacto en el proceso de adquisiciones gestión de materiales. Posiblemente, en el desarrollo del artículo sea necesario utilizar nuevamente la investigación evaluativa con énfasis en la toma de decisiones y no en la evaluación del proceso como se efectuó en la primera fase.

Teniendo en cuenta los resultados de los análisis de datos efectuados en la fase 1 y 2 que correspondieron a la determinación de los principales problemas del Portal de Proveedores, utilizando herramientas evaluativas estadísticas y documentales, en esta fase, mediante la investigación descriptiva, se busca establecer un comportamiento o diagnóstico, aunque este tipo de investigación va enfocada a temas de carácter social, es aplicable a esta fase en la aplicación correlacional, asociando y estableciendo el grado de relación entre factores símiles. En este tipo de investigación no se aportará información sobre las posibles causas de las novedades teniendo en cuenta que fue desarrollada en las fases anteriores.

Finalmente, se investigó documentalmente antecedentes sobre falencias del Portal de Proveedores, analizando informes de auditorías, comunicaciones internas y externas, con el fin de complementar las encuestas realizadas a los proveedores, buscando mejor enfoque a la problemática planteada y corroborar los resultados de las encuestas, debido a que por sí solas pueden ser subjetivas al conceptuar en un momento de tiempo exacto. La documentación permite verificar históricamente fallas sustentadas en diferentes lapsos desde la vigencia 2011 desde la cual se operó en un 100 \% la funcionalidad del sistema.

Una vez realizado el análisis de la problemática del Portal de Proveedores, correlacionando información común del análisis de la información obtenida, en cada una de las fases del trabajo de investigación se clasifican las novedades en dos tipos: en primer lugar, funcionalidad usuario, referente a transacciones y en segundo lugar, el factor de capacitación y manejo del sistema, que corresponde a la pericia del usuario en el manejo del sistema.

\section{Factor funcionalidad usuario}

La principal falencia encontrada en la evaluación efectuada al Portal de Proveedores, diferente a los problemas de conectividad, los cuales no fueron objeto de la investigación, es la ausencia de un campo específico en las cotizaciones, en el cual el proveedor pueda informar a la administración por cuánto tiempo sostiene la propuesta. Es decir la validez de la oferta, aunque al investigar la forma en que se maneja actualmente la fecha de validez, se determinó que en el momento de 
lanzar las peticiones de oferta, se incluye en un campo de datos generales visualizado al momento de cargar la cotización una frase incluida debajo de la forma de pago que indica "Validez de la oferta en días", verificando múltiples cotizaciones el término común solicitado por la FAC para la cotizaciones son 60 días.

Con el análisis de las comunicaciones enviadas por los proveedores evidenciando este tema, se determina que en cada petición de oferta vienen incluidos diferentes números de ítems, para diferentes equipos, inclusive en un proceso se consolidaban compras y reparables haciendo claro que por lo tanto se requiere tener un campo individual por ítem. La ausencia de este dato puede generar que el proveedor no sostenga la oferta, así mismo es una queja generalizada de las diferentes empresas, la demora de la FAC en realizar los procesos contractuales, después de la cotización; al verificar esta información se determinó que los procesos que no se requieren con gran prioridad se pueden tardar hasta 60 días en la adjudicación.

En este caso se pueden plantear dos alternativas para atender este requerimiento. La primera, utilizar el campo de observaciones en cada uno de los ítems para incorporar el dato como se señala en la figura 6, la cual muestra la página del portal de proveedores, opción peticiones de oferta a cotizar, referenciando con un círculo rojo el campo a utilizar, para lo cual se requiere difundir a los proveedores dicha opción, de lo contrario aunque actualmente exista en cada renglón, será subutilizado.

Como segunda alternativa, está la de crear un nuevo campo en la petición de oferta lo cual requiere adelantar una inserción en las tablas actuales, para lo cual es necesario contratar una empresa que realice desarrollos en SAP.

\section{Factor capacitación y retroalimentación}

Aunque el Portal de Proveedores es una herramienta de fácil utilización por cualquier usuario, se ha determinado a lo largo del trabajo de investigación, que los errores presentados frecuentemente por los proveedores, se atribuyen al desconocimiento de las bondades del sistema, las empresas registradas como proveedores, cambian en muchos casos al personal que recibió la capacitación en el Portal y no retroalimentan a otro usuario, lo que genera falta de continuidad en el manejo del sistema.

Se presentan casos tan desconcertantes en el manejo de la información como en el que una empresa manifiesta que no se les envía los ítems a cotizar y que por tal razón la FAC, los ha descartado como proveedor, de lo cual se deduce que confunden el correo de notificación de publicación de petición de oferta, con la utilización de la página Web, lo cual fue manifestado en las diferentes reuniones sostenidas entre proveedores y el personal de la Jefatura de Operaciones Logísticas. Haciendo seguimiento a este caso se concluyó que el proveedor cambio la totalidad de personal que conocía el procedimiento precontractual de la FAC.

En la problemática relacionada con la falta de documentación legal actualizada por parte de los proveedores incorporada en el Portal, se determinó que en el mes de enero de 2012, se lanzaron en vivo cuatro transacciones (ZMM164, ZMM165, ZMM166 Y ZMM170) que permiten crear documentos, alimentar tablas de documentación legal y bloqueo de proveedores, para quienes no cuenten con los documentos actualizados en el Portal, este bloqueo genera que aunque la empresa cuente con las capacidades para suministrar un bien o servicio, no sea invitado.

La incorporación de la documentación legal en el portal de proveedores de las empresas que realizan contratación a través de la Agencia de Compras, es requisito imprescindible para adelantar cualquier proceso, por ejemplo no contar con el certificado de representación legal actualizado, puede traer como consecuencia que quien suscriba el contrato no cuente con la capacidad legal para firmar y por lo tanto, no tenga validez legal el negocio comercial, con las repercusiones administrativas y fiscales derivadas de recursos de presupuesto oficial, así como el impacto generado en el proceso de compras.

La falla en el Portal sobre la incorporación de la documentación legal se debe principalmente a la subutilización del sistema por parte de la Administración, debido a que cuenta con los controles requeridos, adicionalmente el tema de actualización de documentación debe quedar incluido en los ítems a tratar en los programas de capacitación a los usuarios.

El último punto concerniente a la problemática por falta de retroalimentación en el sistema, se debe a la falta de una mecanismo que permita informar al proveedor si el proceso ya fue adjudicado o no, al investigar sobre los antecedentes de esta falencia se determinó que fue manifestado en múltiples ocasiones a la administración por los proveedores, inclusive este tema fue tratado en 
las reuniones efectuadas anualmente con las empresas, por la Jefatura de Operaciones Logísticas, sobre este novedad el Grupo SILOG y la Sección de Sistemas de JOL, han efectuado avances significativos logrando que el desarrollo requerido para realizar esta operación fuera contratado con la empresa Project Core, firma especializada en SAP, con plazo de ejecución diciembre de 2012; con un costo aproximado de USD 800, en el cual se buscó que con una nueva función el proveedor pudiera incorporar el número de petición de oferta cotizada y el sistema muestre el estado de la adquisición, bien sea: en proceso, adjudicado o no adjudicado.

\section{Conclusiones}

La interpretación y aplicación técnica descriptiva indica, que el resultado de la encuesta efectuada a los proveedores evaluando el Portal, requirió segmentación de las diferentes empresas, para establecer que la problemática actual de mayor relevancia en la utilización del sistema, es la falta de retroalimentación de la FAC a los proveedores, al no informar sobre la adjudicación o no de las cotizaciones enviadas. Como segunda falencia se encuentra la falta de capacitación por parte de los diferentes usuarios de la página web, en tercer lugar se encontró la no actualización de la documentación legal exigida para contratar en el sistema y finalmente; la validez de la oferta por cada uno de los ítems, este último factor se refiere a cuánto tiempo sostiene el proveedor su propuesta comercial.

Teniendo en cuenta que el sector aeronáutico, específicamente si en la FAC, no se realiza de forma expedita el proceso de contratación, posiblemente cuando el proveedor reciba el contrato para la firma, este no sea suscrito por la empresa, al no contar con la existencia del elemento ofrecido en la cotización, asimismo el 80 $\%$ de los proveedores no son casas fabricantes ni reparadores, por lo tanto no cuentan con las existencias de los bienes que cotizan.

Como resultado del análisis al Portal de Proveedores efectuado por los Usuarios Internos (Equipo de trabajo de la Subdirección de Adquisiciones), se determinó que la novedad presentada con alta frecuencia y que puede afectar en forma media al proceso de adquisiciones, es la falta de capacitación en el Portal, este diagnóstico se realizó mediante la Técnica de Calificación Relativa y aplicación de la Matriz M4P, con la calificación del personal inmerso en el proceso de gestión de materiales que cuentan con el conocimiento profundo de los requeri- mientos del Portal, así como la experiencia en la utilización del sistema forjado empíricamente en el desempeño laboral desde la puesta en marcha de la página Web.

Lo anterior, permitirá optimizar el desempeño del portal, generando mayor utilización por parte de los proveedores y de los usuarios internos del sistema, alcanzando altos estándares de comunicación con las empresas. Las mejoras en el portal garantizarán la transparencia en la contratación de recursos públicos y al interior de la organización incrementará el grado de alistamiento en aeronaves para desarrollar operaciones aéreas eficaces, cumpliendo la misión de ejercer soberanía del espacio aéreo nacional.

\section{Recomendaciones}

De acuerdo a los resultados es necesario orientar y actualizar en forma continua los niveles de capacitación de la página web, previa consecución de los recursos necesarios y convocatoria confirmando asistencia de los proveedores. Así mismo, se debe dejar constancia de la asistencia del personal mediante levantamiento de acta, la cual debe reposar en dicha Subdirección. Igualmente, se recomienda enfatizar y supervisar las auditorías internas de acompañamiento y auditorías externas para adelantar los procesos de mejora planteados, de igual modo, establecer la exigencia de adelantar todos los procesos precontractuales a través del Portal de Proveedores, con el fin de garantizar la continuidad del sistema permitiendo agilizar el proceso de adquisiciones logísticas.

La propuesta de mejora planteada para el portal de proveedores es viable para la FAC, teniendo en cuenta que se encuentra incluida en el proceso de gestión de materiales, adelantado por la Jefatura de Operaciones Logísticas y esta cuenta con los recursos para efectuar la inversión. La ejecución del plan de acción propuesto para mejorar el portal es menor a un año, en cuanto a su puesta en marcha y reflejo de resultados.

\section{Referencias}

Arese, H. (2003). Matriz de Kraljic. Práctica profesional de negocios internacionales, pp. 150-154.

Ballou, R. (2004). Logística. Administración de la cadena de suministro, pp.86, 96.

Cánez. (2006). Matriz de Kraclic. Estrategias de cadena del Suministro, p.62.

Cárdenas, y otros. (2004). Diagramas. Simulación y análisis de datos, pp.45- 67. 
Carranza. (2004). Historia Logística. Logística y mejores prácticas para Latinoamérica, p. 26.

Contraloría General de la República. (2012). Sistema Integrado de Contratación Estatal. [En línea] Disponible en http://www. sice.gov.co_contratos// Consultado el 15 de junio de 2012.

Departamento de Planeación FAC. (2012). Separata presupuestal vigencia 2012. Bogotá, Colombia: autor.

Fuerza Aérea Colombiana. (2010). Plan estratégico Institucional 2011-2030. Bogotá: autor.

Fuerza Aérea Colombiana. (2011). Cadena del abastecimiento. Revista Aeronáutica, pp.20-22. Bogotá, Colombia: autor.

Hewelt Packard. (2010). Historia del internet. En línea] Disponible en http://www.hp/historia del internet y su llegada a Colombia/2010//. Disponible el 26 de junio de 2012.

Jefatura de Operaciones Logísticas FAC. (2008). Utilización del SAP (Oficio No. 1232-MDN-COFAC-JOL). Bogotá, Colombia: autor. Jefatura de Operaciones Logísticas FAC. (2012) Actividades a desarrollar para la adquisición de bienes y servicios a través de la Agencia de Compras de la Fuerza Aérea Colombiana con sede en Fort Lauderdale, Florida - Estados Unidos de América (Directiva No. 004). Bogotá, Colombia: autor.

Josserand y Monroy. (1951). Efectos de la venta en la legislación foránea, pp. 45, 46.

Legicomex. (2012). Artículos contratación exterior. [En línea] Disponible en http: //www.legicomex.com/normatividadexterior// Consultado el 19 de junio de 2012.

Ley 7a Marco de comercio exterior, publicada en el diario oficial 39631 (1991).

Ley 80 Estatuto de contratación Estatal Congreso República de Colombia (1993).

Ley 87 Normas para el ejercicio del control interno en las entidades y organismos del estado (1993).

Ley 190 Normas para la eficiencia administrativa, la transparencia a través de los sistemas de información y de políticas en el mejoramiento de la gestión pública (1995).

Ministerio de Defensa Nacional. (1956). Modificación del Decreto 0952 de 1953 en cuanto a la comisión de compras en el exterior (Decreto 2409). Bogotá, Colombia: autor.

Ministerio de Defensa Nacional - Oficina Jurídica. (1999). Agencia de compras ejecución de contratos (oficio 0903). Bogotá.

Ministerio de Defensa Nacional. (2007). Implementación e instalación y funcionamiento del sistema SILOG administrado SAP (Resolución Ministerial 3277). Bogotá, Colombia: autor.

Ministerio de Defensa Nacional. (2012). Normatividad contractual. [En línea] Disponible en http://www.mindefensa.gov.co/normatividad// Consultado el 15 de junio de 2012.

Ministerio de Relaciones Exteriores. (2012). Reglamentación del Tratado de Libre Comercio entre Colombia y Estados Unidos de Norteamérica (Decreto 993). Bogotá, Colombia: autor.

Presidencia de la República. (1953). Por el cual se crea una comisión permanente de compras en el exterior (Decreto 0952). Bogotá, Colombia: autor.

PROQUEST. (2012). Adquisiciones militares. Consultado en la plataforma IMA 2012, desde http://www.ima.edu.co
SILOG. (2010). Guía del Usuario del Portal de Proveedores. Bogotá, Colombia: autor.

SILOG. (2012). Portal de Proveedores. [En línea] Disponible en http://www. jolacofa.fac.mil.co/irj/portal Consultado el 22 de junio de 2012.

Pérez, R. I. (2012). Priorización de problemas en ambientes de seguridad operacional y mantenimiento de aeronaves: Dos aplicaciones. Rev. Ciencia y Poder Aéreo. 7(ISSN 1909-7057), pp. 08-18. Bogotá, Colombia: Instituto Militar Aeronáutico, Fuerza Aérea Colombiana.

Peña, S. M. (2012). Propuesta de mejora al proceso de adquisiciones logísticas aeronáuticas a través del portal de proveedores de la agencia de compras FAC. Trabajo de grado para optar el título de Especialista en Logística Aeronáutica, Bogotá, Colombia: Instituto Militar Aeronáutico. Fuerza Aérea Colombiana.

Zabala. (2009). Referencias. Guía a la redacción en el estilo APA, pp. $5-8$.
Para citar este artículo:

Peña, S., y Estrada, E. (2014). Diagnóstico del proceso de adquisiciones logísticas aeronáuticas a través del portal de proveedores de la agencia de compras FAC. Ciencia y Poder Aéreo, Vol. 9 (1). Pp. 43-53 